\title{
Evaluación de un nomograma predictivo de éxito de la litotricia extracorpórea por ondas de choque en el tratamiento de la urolitiasis
}

\section{Evaluation of a nomogram for predicting successful kidney stone treatment through extracorporeal shock wave lithotripsy}

Santiago Arturo Avendaño-Bastidas, ${ }^{1}$ Manuel Alcaraz-Gutiérrez, ${ }^{2}$ Javier Medrano-Sánchez, ${ }^{1}$ Carlos RíosMelgarejo, ${ }^{1}$ Julio Guillén--Rodríguez, ${ }^{1}$ Enrique Pulido-Contreras, ${ }^{1}$ Miguel Ángel García-Padilla ${ }^{1}$

\begin{abstract}
Resumen
OBJETIVO: Analizar el valor predictivo de un nomograma, identificar las variables predictoras de tasa libre de cálculos y evaluar el estado actual de la litotricia extracorpórea por ondas de choque como tratamiento de la litiasis renal.

MATERIALES Y MÉTODOS: Estudio prospectivo efectuado en pacientes con diagnóstico de litiasis renal y tomografía computada (TAC) tres semanas después de la última sesión de litotrisia extracorpórea. Determinación del estado libre de cálculos a las 3 semanas postratamiento. Evaluar el rendimiento pronóstico del nomograma de Kim vs TAC para determinar la predicción del estado libre de cálculos. Se realizó estadística bayesiana para calcular la prevalencia de litiasis después de la prueba y se analizó con curva ROC para determinar el área bajo la curva de todos los valores; además, reporte del IC95\%. RESULTADOS: Se registraron 110 pacientes, la eficacia general de la litotricia extracorpórea fue de $69 \%$. El análisis del nomograma mostró un área bajo la curva de 0.627 . El número y diámetro máximo de los cálculos mostraron diferencias estadísticamente significativas en la tasa libre de cálculos. Al evaluar otras variables se determinó que el volumen y las unidades Hounsfield también fueron predictivas del resultado final del tratamiento.
\end{abstract}

CONCLUSIONES: La litotricia extracorpórea por ondas de choque representa un tratamiento aún efectivo en pacientes con litiasis renal. Con las variables predictivas identificadas puede efectuarse una mejor selección de pacientes para practicar esta técnica. PALABRAS CLAVE: Nomograma; litoricia extracorpórea; litiasis renal.

\section{Abstract}

OBJECTIVE: To analyze the predictive performance of a nomogram and identify other predictive variables of stone-free rate, as well as to evaluate the current status of extracorporeal shock wave lithotripsy in the treatment of kidney stones.

MATERIALS AND METHODS: The prospective evaluation of the Kim nomogram as a predictive tool for stone-free rate after extracorporeal shock wave lithotripsy in patients previously assessed through computed tomography was carried out. Stone status was determined 3 weeks after treatment. The predictive yield of the Kim nomogram versus the reference study (computed tomography) was evaluated for predicting stone-free status. Bayesian statistics were performed to calculate the prevalence of stones after the test and the ROC curve analysis was carried out to determine the area under the curve for all values, as well as to report the $95 \% \mathrm{Cl}$.

\author{
${ }^{1}$ Adscrito al servicio de Urología. \\ ${ }^{2}$ Residente de Urología. \\ Unidad Médica de Alta Especialidad 1, \\ Centro Médico Nacional del Bajío, León, \\ Guanajuato.
}

Recibido: marzo 2017

Aceptado: julio 2018

Correspondencia

Santiago Arturo Avendaño Bastidas uromane8903@gmail.com

Manuel Alcaraz Gutiérrez uromane8903@gmail.com

Este artículo debe citarse como Avendaño-Bastidas AS, Alcaraz-Gutiérrez $\mathrm{M}$, Medrano-Sánchez J, RíosMelgarejo C, Guillén-Rodríguez J, Pulido-Contreras E, García-Padilla MA. Reporte de un caso. Evaluación de un nomograma predictivo de éxito de la litotricia extracorpórea por ondas de choque en el tratamiento de la urolitiasis. Rev Mex Urol. 2018 julioagosto;78(4):273-282.

DOI: https://doi.org/10.24245/revmexurol.v78i4.2068 
RESULTS: A total of 110 patients were included in the study. Extracorporeal lithotripsy showed a general efficacy of $69 \%$ and the nomogram analysis produced an area under the curve of 0.627 . The number of stones and the maximum diameter were statistically significant variables for achieving the stone-free rate. Stone volume and Hounsfield units were also predictive in the final treatment result.

CONCLUSIONS: Extracorporeal shock wave lithotripsy is still an effective treatment for kidney stones. A better selection of patients that are candidates for said treatment can be made utilizing the predictive variables identified.

KEYWORDS: Nomogram; Extracorporeal lithotripsy; Kidney stone.

\section{ANTECEDENTES}

La prevalencia de litiasis renal varía de 1 a $20 \%$ en la población general, según el sexo, edad, raza y área geográfica. ${ }^{1}$ De acuerdo con datos recientes, la prevalencia en hombres es de 10.6\% y en mujeres de $7.1 \%$. La incidencia más alta se registra en pacientes de 40 a 60 años de edad. En orden de prevalencia, afecta a caucásicos, hispanos, asiáticos y africanos. La distribución geográfica se relaciona con factores de riesgo ambiental, con mayor prevalencia en áreas con climas cálidos, áridos o secos. ${ }^{2}$ En México existen pocos estudios epidemiológicos al respecto; por ejemplo, Otero y sus colaboradores reportan $13 \%$ de las hospitalizaciones en el IMSS, ${ }^{3}$ pero en el Hospital General de México se ha calculado una incidencia de 24 por 10,000 habitantes; además, se estima que entre 10 y $12 \%$ de todos los individuos padecerá urolitiasis en algún momento de su vida. ${ }^{4}$

El primer tratamiento exitoso mediante litotricia extracorpórea por ondas de choque se efectuó en Alemania, en 1980, por el Dr. Christian Chaussy, quien utilizó un litotriptor Dornier HM1. En Estados Unidos Lingeman usó un litotriptor Dornier HM3 en el primer procedimiento. ${ }^{5}$ Desde entonces y gracias al desarrollo de equipos más efectivos y modernos se efectúan procedimientos quirúrgicos abiertos por técnicas endourológicas, mínimamente invasivas, con litotricia extracorpórea por ondas de choque.
Entre los factores que afectan los resultados de la litotricia extracorpórea por ondas de choque se encuentran: algunos tipos de minerales resistentes a la fragmentación (cistina homogénea, brushita, algunos cálculos de oxalato de calcio monohidratado), ${ }^{6}$ anatomía y alteraciones renales (riñón en herradura, divertículos de los cálices, ectopia renal), e hidronefrosis severa. ${ }^{2}$ La eficacia de la litotricia extracorpórea por ondas de choque puede afectarse por el IMC; algunos estudios indican resultados poco satisfactorios cuando la distancia entre el cálculo y la piel es mayor de $10 \mathrm{~cm} \cdot{ }^{7-9} \mathrm{~A}$ mayor densidad menor efectividad de la litotricia extracorpórea. Diversas investigaciones han demostrado el valor predictivo de la densidad del cálculo y se han establecido, incluso, 1000 UH como punto de corte para el éxito del tratamiento; sin embargo, dos estudios establecen que el valor mayor de $900 \mathrm{UH}$ se relaciona con fracaso del tratamiento. ${ }^{1,7,9,10}$ En cuanto al tamaño del cálculo, se han evaluado múltiples medidas que tratan de establecerse como factor predictor. Un estudio demostró que el volumen del cálculo representa un predictor más preciso que el diámetro para pronosticar el éxito de la litotricia extracorpórea. ${ }^{11}$

Investigaciones actuales señalan disminución de la efectividad de la litotricia extracorpórea por ondas de choque (LEOCH), mientras que la de ureterorrenoscopia se ha incrementado en cuanto a tasa libre de cálculos. ${ }^{12}$ La LEOCH es 
efectiva en pacientes con cálculos menores de 20 mm, no así en los que se encuentran en el cáliz inferior. ${ }^{13,14}$ La tasa libre de cálculos para LEOCH localizados en el cáliz inferior es de 25-95\%. Los pacientes con cálculos renales mayores de 20 mm, intervenidos con litotricia extracorpórea por ondas de choque suelen requerir una nueva reintervención; por tanto, debe considerarse el tratamiento con nefrolitotomía percutánea. ${ }^{15}$ Algunos estudios demuestran ventajas de la ureterorrenoscopia versus litotricia extracorpórea; sin embargo, requieren métodos más invasivos. Los pacientes con cálculos de hasta $3 \mathrm{~cm}$ pueden tratarse con ureterorrenoscopia o litotricia con láser, pero a mediano o largo plazo demandarán un nuevo procedimiento. ${ }^{16-19}$

La disminución en la eficacia de la litotricia extracorpórea por ondas de choque ha generado dudas respecto de su costo-beneficio sobre otras opciones, principalmente la ureterorrenoscopia con litotricia láser y nefrolitotomía percutánea. Una revisión actual de Cochrane no reportó diferencias estadísticamente significativas de la litotricia extracorpórea por ondas de choque en cuanto a tasa libre de cálculos; sin embargo, la nefrolitotomía percutánea y ureterorrenoscopia fueron superiores en: disminución del periodo de tratamiento, costo-beneficio, complicaciones y tiempo de estancia hospitalaria, ${ }^{20}$ estos últimos considerados factores importantes para la reincorporación de la población en edad productiva.

El uso de este nomograma como herramienta pronóstica permite orientar en la toma de decisiones terapéuticas y costo-beneficio de la litotricia extracorpórea por ondas de choque, con base en la evaluación de los resultados finales y de esta manera definir qué pacientes se beneficiarán de la LEOCH versus las otras opciones de tratamiento.

Hasta la fecha no existen métodos estandarizados que pronostiquen la efectividad de la LEOCH en el tratamiento de la litiasis renal. Kanao y su grupo, en un estudio con 435 pacientes, utilizaron un nomograma para evaluar la efectividad del tratamiento en cada sesión después de 3 meses. Entre sus resultados indicaron diferencias significativas en la longitud, ubicación y número de cálculos; ${ }^{22}$ sin embargo, utilizaron una radiografía simple de abdomen y urografía excretora para la evaluación pre-LEOCH y durante el seguimiento dejaron de lado la información útil que ofrece la tomografía computada, que hasta la fecha representa el estudio de referencia para el análisis de la urolitiasis. ${ }^{23}$ Otro estudio retrospectivo que evaluó los cálculos renales o ureterales solitarios, de $20 \mathrm{~mm}$ o menores, reportó como predictores significativos las variables de: edad, tamaño del cálculo y distancia cálculo-piel; no obstante, la radiografía simple de abdomen, solicitada 3 meses posteriores a una única sesión de litotricia extracorpórea, sin utilizar variables continuas, demostró una tasa de éxito para eliminar cálculos renales de $70.2 \%$, con discriminación predictiva del área bajo la curva de $0.75 .{ }^{24}$ Un estudio que evaluó un nomograma con análisis multivariado mostró como factores predictivos independientes para estado libre de cálculos las variables tomográficas en cuanto al tamaño, unidades Hounsfield (UH) y distancia cálculo-piel, con área bajo la curva estimada de 0.760 , demostrando una alta precisión predictiva; sin embargo, solo fue efectivo para cálculos solitarios ureterales del tercio proximal, dejando fuera los cálculos renales. ${ }^{25}$ En 2015, Timothy y sus colaboradores crearon un sistema de puntuación llamado "triple D", debido a las variables tomográficas que incorpora: densidad y volumen del cálculo, y distancia cálculo-piel como método predictor de éxito de la litotricia extracorpórea por ondas de choque. Según el valor calculado para cada variable, se clasificó en 4 grupos y se correlacionó cada uno con la tasa libre de cálculo, respectivamente, lo que resultó en área bajo la curva de $0.8338 ;{ }^{26} \sin$ embargo, en los estudios de control no consideraron la 
tomografía, sino el ultrasonido y la radiografía después del tratamiento para concluir el estado libre de cálculos.

Diversos estudios seleccionan el nomograma de Kim para su validación, por tratarse de un método reciente y con mayor número de variables, además considera características tomográficas e información clínica del paciente, lo que demuestra un valor pronóstico adecuado. ${ }^{27}$ En la población mexicana no existe ningún estudio que analice las variables pronósticas para tasa libre de cálculos por litotricia extracorpórea por ondas de choque, por lo que se requiere una evaluación actualizada en este contexto.

Con base en lo anterior, el objetivo de este estudio fue: analizar el valor predictivo de un nomograma, identificar las variables predictoras de tasa libre de cálculos y evaluar el estado actual de la LEOCH como tratamiento para la litiasis renal.

\section{MATERIALES Y MÉTODOS}

Estudio prospectivo, llevado a cabo en el servicio de Urología de la Unidad Médica de Alta Especialidad 1, Centro Médico Nacional del Bajío, León, Guanajuato, entre mayo y noviembre de 2017. Se seleccionaron pacientes con diagnóstico de litiasis renal y que contaran con tomografía computada después de 3 semanas de haber concluido la última sesión de tratamiento con LEOCH. Los pacientes se asignaron a 2 grupos: uno correspondiente a libre de cálculos y el otro con litiasis residual. Se trata de un estudio para evaluar una prueba diagnóstica para la validación del nomograma de Kim, con inclusión de características clínicas y tomográficas que se analizaron antes de iniciar el tratamiento.

Criterios de inclusión: pacientes mayores de 18 años, de uno y otro sexo, con diagnóstico de litiasis renal confirmada por tomografía com- putada, ingresados al servicio de Urología para intervenirse de litotricia extracorpórea por ondas de choque. Criterios de exclusión: pacientes con coagulopatías, marcapasos, infección de vías urinarias no tratadas, mujeres embarazadas, pacientes que por dificultades técnicas no completaron el tratamiento de litotricia extracorpórea y quienes abandonaron el protocolo y seguimiento o no contaron con tomografía de control posterior al término del tratamiento. El tamaño de la muestra se cálculo con base en la fórmula de Martín Andrés y Luna del Castillo (2004), tomando en cuenta 250 tratamientos de litotricia extracorpórea por ondas de choque al año en la unidad médica; la bibliografía reporta sensibilidad de $92 \%$ de la tomografía computada en la evaluación del estado libre de cálculos (cálculos residuales iguales o menores de 4 $\mathrm{mm}$ ) y detecta una diferencia igual o menor de $10 \%$ en la sensibilidad del nomograma de Kim, comparado con el estudio de referencia y con potencia de $20 \%$. De acuerdo con la fórmula se obtuvieron 110 pacientes, con valor alfa de $5 \%$ para dos colas.

El nomograma incluye 6 variables: 1) sexo, 2) localización del cálculo (cortes axial y coronal de la tomografía; definido según correspondan en el cáliz superior, medio e inferior, pelvis renal o uréter proximal), 3) número de cálculos (detectados por tomografía computada), 4) diámetro máximo del cálculo, 5) grado de hidronefrosis mediante estudio tomográfico (clasificándolo en 5 grados: $0=$ sin hidronefrosis, $1=$ pelvis renal ligeramente dilatada sin ectasia calicial, 2 = pelvis renal moderadamente dilatada con ectasia calicial, 3 = pelvis renal grande, cálices dilatados y parénquima renal normal y $4=$ pelvis renal grande, cálices grandes y dilatados, además de adelgazamiento del parénquima renal) y 6) unidades Hounsfield.

Además de las variables del nomograma de Kim se estudiaron las siguientes: edad, lado 
afectado, litiasis (primer episodio o recidiva), litiasis residual posterior al tratamiento inicial (cirugía abierta, nefrolitotomía percutánea, ureterorrenoscopia flexible), colocación de catéter doble J, volumen del cálculo y distancia cálculo-piel.

A cada variable del nomograma se le asignó un puntaje determinado por la escala del nomograma de Kim; el total de puntos representó la probabilidad de tasa libre de cálculos al final del tratamiento. El estudio tomográfico se efectuó antes de llevar a cabo el tratamiento por el urólogo y el técnico radiólogo encargados del mismo, sin que hubiera influencia en la decisión del número, potencia, o ambos, de las sesiones realizadas en la investigación. Se solicitó la tomografía computada simple de abdomen a la tercer semana de haber concluido el tratamiento, donde se evaluó el estado litiásico, considerando "tasa libre de cálculos" a la ausencia o coexistencia de alguno de éstos, menores de $3 \mathrm{~mm}$.

\section{Análisis estadístico}

Se realizó estadística descriptiva para conocer la distribución de las variables que cumplieron con los criterios de normalidad, según la prueba de Kolmogorov; se reportaron como media y desviación estándar las que no lo cumplieron, y como mediana y rango intercuartilar $25-75 \%$. Las variables cualitativas se reportaron como frecuencia y porcentaje. Se evaluó el rendimiento pronóstico del nomograma de Kim versus el estudio de referencia (tomografía computada) para determinar la predicción del estado libre de cálculos. Se realizó estadística bayesiana para calcular la prevalencia después de la prueba y se analizó con curva ROC para determinar el área bajo la curva de todos estos valores, además, se reportó el intervalo de confianza de 95\% (IC95\%).

\section{RESULTADOS}

De los 110 pacientes seleccionados en el estudio, 61 (55.4\%) correspondieron al sexo femenino y $49(44.6 \%)$ al masculino ( $p=0.157)$. La localización más frecuente de los cálculos fue en el cáliz inferior $41(37.2 \%)$, pelvis $27(24.5 \%)$, cáliz medio 23 (20.9\%), uréter proximal 11 (10\%) y cáliz superior 8 (7.2\%), que representó una $p=0.811$, sin diferencia significativa entre el grupo libre de cálculos versus litiasis residual. En cuanto al número de cálculos, se encontraron 75 (68.1\%) pacientes con 1 cálculo, 20 (18.1\%) con $2 ; 9$ (8.1\%) con 3; 1 (0.9\%) con 4; 3 (2.7\%) con 5 y 1 ( $0.9 \%)$ con 6 cálculos; no se registraron casos con 7 cálculos, pero se registró 1 (0.9\%) paciente con 8 cálculos, lo que demostró una significación estadística $(p=0.001)$ entre ambos grupos. Respecto del diámetro máximo del cálculo, en los pacientes libres de cálculos fue de $9.68 \pm 3.27 \mathrm{~cm}$ versus cálculos residuales de $11.97 \pm 4.39 \mathrm{~cm}(p=0.003)$. Solo se registró $1(2.8 \%)$ caso de hidronefrosis $(p=0.150)$. En cuanto a la densidad se encontró una media de $788 \pm 349$ UH en el grupo libre de cálculos y de $930 \pm 447$ UH en el de litiasis residual $(p=$ 0.072). Cuadro 1

En relación con las variables que se estudiaron y no se incluyeron en el nomograma de Kim, sólo el volumen litiásico mostró significación estadística entre los grupos ( $p=0.016)$ (Cuadro 2). Al evaluar la densidad del cálculo, se establecieron como punto de corte 900 y 1000 UH, este último con diferencia estadísticamente significativa en la densidad igual o menor de 1000 UH para tasa libre de cálculo. Cuadro 3

Tomando en cuenta la distancia cálculo-piel de $10 \mathrm{~cm}$ como valor de corte para la tasa libre de cálculo, no se encontró significación estadística $(p=0.82)$. Cuadro 4

En lo que a número de sesiones y su relación con el resultado al final del tratamiento respecta, se observó que después de la cuarta sesión disminuye la tasa libre de cálculo. Cuadro 5

Al analizar con estadística bayesiana el rendimiento diagnóstico del nomograma de Kim 
Cuadro 1. Análisis de las variables del nomograma de Kim

\begin{tabular}{|c|c|c|c|}
\hline Variable & $\begin{array}{l}\text { Libre de } \\
\text { cálculos } \\
(n=74)\end{array}$ & $\begin{array}{l}\text { Litiasis } \\
\text { residual } \\
(n=36)\end{array}$ & $\mathbf{p}$ \\
\hline \multicolumn{4}{|l|}{ Sexo } \\
\hline $\begin{array}{l}\text { Femenino } \\
\text { Masculino }\end{array}$ & $\begin{array}{l}45(60.8 \%) \\
29(39.2 \%)\end{array}$ & $\begin{array}{l}16(44.4 \%) \\
20(55.6 \%)\end{array}$ & $\mathrm{p}={ }_{*} 0.157$ \\
\hline \multicolumn{4}{|l|}{ Localización } \\
\hline $\begin{array}{l}\text { Cáliz inferior } \\
\text { Cáliz medio } \\
\text { Cáliz superior } \\
\text { Pelvis } \\
\text { Uréter proximal }\end{array}$ & $\begin{array}{c}26(35.1 \%) \\
16(21.6 \%) \\
5(6.8 \%) \\
18(24.5 \%) \\
9(12.2 \%)\end{array}$ & $\begin{array}{c}15(41.7 \%) \\
7(19.4 \%) \\
3(8.3 \%) \\
9(25 \%) \\
2(5.6 \%)\end{array}$ & $\mathrm{p}=\underset{*}{0.811}$ \\
\hline \multicolumn{4}{|l|}{ Número de cálculos } \\
\hline $\begin{array}{l}1 \\
2 \\
3 \\
4 \\
5 \\
6 \\
7 \\
8\end{array}$ & $\begin{array}{c}51(68.9 \%) \\
17(23 \%) \\
1(1.4 \%) \\
1(1.4 \%) \\
3(4.1 \%) \\
- \\
- \\
1(1.4 \%)\end{array}$ & $\begin{array}{c}24(66.7 \%) \\
3(8.3 \%) \\
8(22.2 \%) \\
- \\
- \\
1(2.8 \%) \\
- \\
-\end{array}$ & \multirow{2}{*}{$\begin{array}{c}\mathrm{p}=0.001 \\
* \\
\mathrm{p}=\underset{* *}{*} 0.003\end{array}$} \\
\hline Diámetro máximo & $\begin{array}{c}9.68 \pm \\
3.27 \mathrm{~mm}\end{array}$ & $\begin{array}{c}11.97 \pm \\
4.39 \mathrm{~mm}\end{array}$ & \\
\hline \multicolumn{4}{|c|}{ Grado de hidronefrosis } \\
\hline $\begin{array}{l}1 \\
2 \\
3 \\
4\end{array}$ & $\begin{array}{l}- \\
- \\
- \\
-\end{array}$ & $\begin{array}{c}1(2.8 \%) \\
- \\
- \\
-\end{array}$ & $\begin{array}{c}\mathrm{p}=0.150 \\
*\end{array}$ \\
\hline $\begin{array}{l}\text { Unidades } \\
\text { Hounsfield }\end{array}$ & $788 \pm 349$ & $930 \pm 447$ & $\begin{array}{c}\mathrm{p}=0.072 \\
* *\end{array}$ \\
\hline
\end{tabular}

* La variable se reporta como frecuencia y porcentaje, y la comparación entre grupos se realiza con la prueba exacta de Fisher. ** Variable con distribución normal, se reporta como media y desviación estándar, la comparación entre grupos se realizó a través de una prueba t de Student para grupos independientes.

versus tomografía computada para tasa libre cálculos, se encontró que con el puntaje de 75.5, el pico máximo de sensibilidad del nomograma fue 0.986 y especificidad de 0.861 ; sin embargo, los grupos correspondientes a tasa libre de cálculos y con litiasis residual tuvieron el mismo puntaje y el área bajo la curva del nomograma fue de 0.627 (Figura 1).
Cuadro 2. Análisis de las variables no incluidas en el nomograma de Kim

\begin{tabular}{|c|c|c|c|}
\hline Variable & $\begin{array}{l}\text { Libre de } \\
\text { cálculos } \\
(n=74)\end{array}$ & $\begin{array}{l}\text { Litiasis } \\
\text { residual } \\
(n=36)\end{array}$ & p \\
\hline Edad & $\begin{array}{c}48.24 \\
\pm 12.53\end{array}$ & $\begin{array}{l}45.08 \pm \\
11.17\end{array}$ & $\mathrm{p}=\underset{* *}{0.179}$ \\
\hline $\begin{array}{l}\text { Lado afectado } \\
\text { Bilateral } \\
\text { Derecho } \\
\text { Izquierdo }\end{array}$ & $\begin{array}{c}1(1.4 \%) \\
31(41.9 \%) \\
42(56.8)\end{array}$ & $\begin{array}{c}0(0 \%) \\
18(50 \%) \\
18(50 \%)\end{array}$ & $p=509 *$ \\
\hline $\begin{array}{l}\text { Primer episodio de } \\
\text { litiasis renal }\end{array}$ & $63(85.1 \%)$ & $\begin{array}{c}31 \\
(86.1 \%)\end{array}$ & $\begin{array}{c}\mathrm{p}= \\
0.891 \\
*\end{array}$ \\
\hline $\begin{array}{l}\text { Recidiva de litiasis } \\
\text { renal }\end{array}$ & $11(14.9 \%)$ & $5(13.9 \%)$ & $\begin{array}{c}\mathrm{p}=0.892 \\
*\end{array}$ \\
\hline $\begin{array}{l}\text { Litiasis residual } \\
\text { posterior a un } \\
\text { tratamiento previo }\end{array}$ & $9(12.2 \%)$ & $6(16.7 \%)$ & $p=524^{*}$ \\
\hline $\begin{array}{l}\text { Colocación de } \\
\text { catéter doble J }\end{array}$ & $25(33.8 \%)$ & 14 (38.9) & $\begin{array}{c}\mathrm{p}=0.754 \\
*\end{array}$ \\
\hline Volumen & $\begin{array}{c}294(150- \\
524)\end{array}$ & $\begin{array}{c}524(237- \\
1036)\end{array}$ & $\mathrm{p}=\underset{* * *}{0.016}$ \\
\hline $\begin{array}{l}\text { Distancia } \\
\text { cálculo-piel }\end{array}$ & $\begin{array}{c}97.66 \pm \\
19.3 \mathrm{~mm}\end{array}$ & $\begin{array}{l}94.97 \pm \\
18.1 \mathrm{~mm}\end{array}$ & $\begin{array}{c}\mathrm{p}= \\
* *\end{array}$ \\
\hline
\end{tabular}

* La variable se reporta como frecuencia y porcentaje, y la comparación entre grupos con una prueba exacta de Fisher. ${ }^{* *}$ Variable con distribución normal: se reporta como media y desviación estándar, la comparación entre grupos se realizó con la t de Student para grupos independientes. ${ }^{* * *}$ Variable con distribución no normal, se reporta como mediana y percentil 25-75\%, la comparación entre los grupos se realizó mediante la prueba $U$ de Mann-Whitney.

Cuadro 3. Densidad del cálculo, tomando como punto de corte $1000 \mathrm{UH}$

\begin{tabular}{l|c|c|c} 
Variable & $>\mathbf{1 0 0 0} \mathbf{U H}$ & $\mathbf{5 1 0 0 0} \mathbf{U H}$ & $\mathbf{p}$ \\
$\begin{array}{l}\text { Pacientes libres } \\
\text { de cálculos }\end{array}$ & $16(21.6 \%)$ & $58(78.3 \%)$ & \\
$\begin{array}{l}\text { Pacientes con } \\
\text { litiasis residual }\end{array}$ & $15(41.6 \%)$ & $21(58.3 \%)$ & $\mathrm{p}=0.0413$
\end{tabular}

La comparación entre grupos se realizó con una prueba exacta de Fisher.

Cuadro 4. Tasa libre de cálculos, tomando la distancia cálculo-piel con valor de corte de $10 \mathrm{~cm}$

\begin{tabular}{l|c|c|c} 
Variable & $>\mathbf{1 0} \mathbf{c m}$ & $\leq \mathbf{1 0} \mathbf{~ c m}$ & $\mathbf{p}$ \\
$\begin{array}{l}\text { Pacientes libres de } \\
\text { cálculos }\end{array}$ & $25(34.2 \%)$ & $48(65.7 \%)$ & $\mathrm{p}=0.82$ \\
$\begin{array}{l}\text { Pacientes con } \\
\text { litiasis residual }\end{array}$ & $12(34.2 \%)$ & $23(65.7 \%)$ &
\end{tabular}

La comparación entre grupos se realizó con la prueba exacta de Fisher. 


\section{DISCUSIÓN}

En la práctica médica los nomogramas son útiles para la toma de decisiones. En cuanto a la urolitiasis, los nomogramas aprobados y validados

Cuadro 5. Relación entre el número de sesiones versus resultado al final del tratamiento

\begin{tabular}{|l|c|c|}
\hline Sesión & $\begin{array}{c}\text { Litiasis residual } \\
(\mathbf{n = 3 6 )}\end{array}$ & $\begin{array}{c}\text { Libre de cálculos } \\
(\mathbf{n = 7 4 )}\end{array}$ \\
\hline 1 & $4(11.1 \%)$ & $28(37.8 \%)$ \\
\hline 2 & $8(22.2 \%)$ & $21(28.3 \%)$ \\
\hline 3 & $8(22.2 \%)$ & $10(13.5 \%)$ \\
\hline 4 & $7(19.4 \%)$ & $11(14.8 \%)$ \\
\hline 5 & $8(22.2 \%)$ & $2(2.7 \%)$ \\
\hline 6 & $1(2.7 \%)$ & $1(1.3 \%)$ \\
\hline 7 & - & $1(1.3 \%)$ \\
\hline
\end{tabular}

A partir de la quinta sesión disminuye la tasa libre de cálculo.

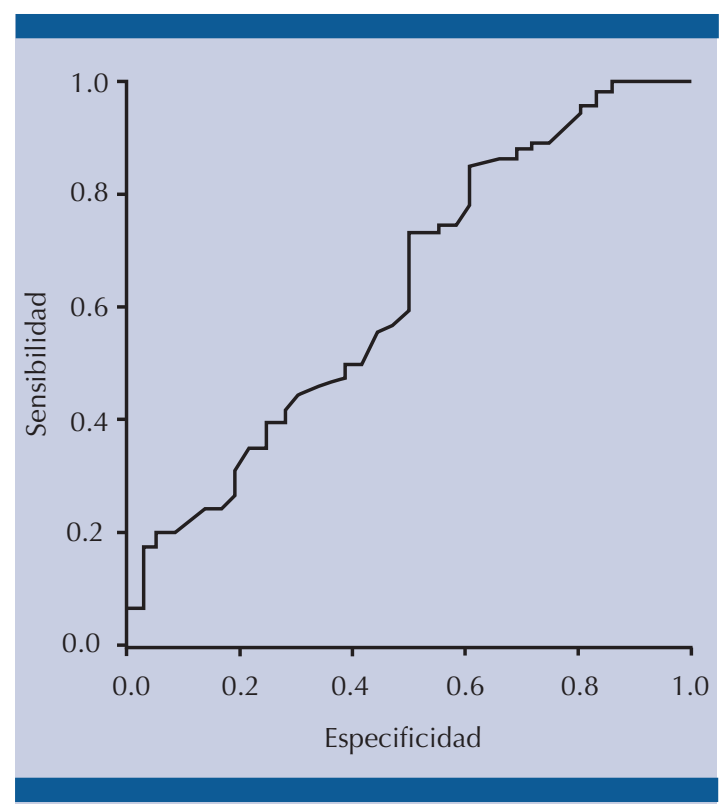

Figura 1. Curva ROC de sensibilidad y especificidad.

se relacionan, principalmente, con la nefrolitotomía percutánea; ${ }^{21}$ sin embargo, la litotricia extracorpórea por ondas de choque no cuenta con nomogramas estandarizados por la Sociedad Urológica Internacional y los pocos que existen no utilizan la mayor parte de la información obtenida de la tomografía computada, se obtienen variables heterogéneas y el control posterior al tratamiento suele evaluarse con radiografía simple de abdomen, que puede proporcionar mayor número de falsos negativos para determinar y establecer la tasa libre de cálculos.

En este estudio se consideró el recientemente formulado nomograma de Kim con mayor número de variables desarrolladas a partir de 3028 casos. $^{27}$ El nomograma mostró, en el análisis univariado, que el sexo, localización del cálculo, número y diámetro máximo fueron predictores significativos para establecer la tasa libre de cálculos. Posteriormente, en otro análisis se agregaron las unidades Hounsfield y el grado de hidronefrosis, que se evaluaron en un modelo de regresión logística multivariado, donde a excepción del sexo, se asociaron significativamente con la tasa libre de cálculos. En el estudio del nomograma de Kim se observó un área bajo la curva de 0.71 , lo que representa una discriminación y predicción aceptable.

La efectividad general del nomograma para establecer la tasa libre de cálculos mediante litotricia extracorpórea por ondas de choque correspondió a $69 \%$, cifra menor al estudio de Wiesenthal y su grupo, quienes señalaron $70.2 \%$, pero mayor al de Wang y Timothy y sus colaboradores, con 52.5 y $59.5 \%$, respectivamente. 10,24,26 $^{2}$

Las variables con significación estadística fueron el número de cálculos ( $p=0.001)$, que a partir de 2 tuvo repercusión negativa en la tasa libre de cálculos, y su tamaño ( $p=0.003$ ), con asociación significativa cuando correspondió a $9.68 \pm 3.27 \mathrm{~mm}$. Las unidades Hounsfield (aunque reportaron buen puntaje, $p=0.072$ ) y la localización del cálculo no fueron variables significativas, quizá debido a la preselección de 
casos en la consulta externa, porque al observar una localización con anatomía poco favorable y densidad muy alta no se consideran ideales para realizar la litotricia extracorpórea por ondas de choque como terapia inicial. Otros estudios también señalan que la localización del cálculo no tiene valor estadísticamente significativo..$^{24,26}$ Una variable que considera el nomograma de Kim es el grado de hidronefrosis, que representa un factor en contra para establecer la tasa libre de cálculos. En este estudio no evaluamos dicha variable, porque solo encontramos un paciente con hidronefrosis grado 1. De acuerdo con ese dato, este tipo de pacientes no son ideales, de primera instancia, para practicar la litotricia extracorpórea por ondas de choque en nuestro servicio.

De las variables que no formaron parte del nomograma, pero que se evaluaron en este estudio, solo el volumen del cálculo $(p=0.016)$ resultó estadísticamente significativo. La edad, lado afectado, primer episodio de litiasis renal o evento recurrente, litiasis posterior a un tratamiento previo, colocación o no de catéter doble J y distancia cálculo-piel no fueron estadísticamente significativas. En una evaluación secundaria, la distancia cálculo-piel con valor de corte de 10 $\mathrm{cm}$ no mostró precisión predictiva de tasa libre de cálculos $(p=0.82)$. Al igual que el estudio de Kim et al, y Niwa y sus colaboradores expusieron un nomograma, donde el tamaño del cálculo, densidad y distancia cálculo-piel representaron factores predictivos para establecer la tasa libre de cálculos; sin embargo, solo reportaron cálculos únicos localizados en el uréter proximal y utilizaron radiografías simples de abdomen como estudios de control posterior al tratamiento, mostrando un área bajo la curva por arriba de 0.7. ${ }^{25,27}$ Un estudio retrospectivo reportó como variables estadísticamente significativas para tasa libre de cálculos: volumen, distancia cálculo-piel y unidades Hounsfield. Y en relación con esta última, definieron que a partir de $12.4 \mathrm{~cm}$ corres- pondió al grupo con litiasis residual. En nuestro estudio registramos 7 pacientes con distancia cálculo-piel mayor de $12.4 \mathrm{~cm}$, principalmente en el grupo de tasa libre de cálculos. Por tanto, no representó un factor importante; sin embargo, se requieren estudios adicionales en este grupo de pacientes, incluso para compararlo con el índice de masa corporal. ${ }^{26}$

Al evaluar la densidad del cálculo mediante tomografía computada se estableció un punto de corte de 900 UH y se encontró disminución de la tasa libre de cálculos con más de 900 UH, con diferencia estadísticamente significativa $(p=0.04)$. Otro resultado secundario fue que posterior a la cuarta sesión de tratamiento, la tasa libre de cálculos no fue satisfactoria para cualquier variable.

La evaluación del nomograma de este estudio mostró un puntaje de 75.5 como el límite máximo de sensibilidad y especificidad; de esta forma, la sensibilidad correspondió a 0.986 y especificidad 0.861 ; sin embargo, ambos grupos (tasa libre de cálculos versus litiasis residual) registraron ese puntaje y el área bajo la curva del nomograma fue de 0.627 , lo que correspondió a un valor inferior al estudio de Kim y su grupo; por tanto, no mostró utilidad como prueba predictiva para establecer la tasa libre de cálculos de nuestro análisis, quizá porque en nuestra población solo 2 de las 6 variables del nomograma fueron estadísticamente significativas (número y diámetro máximo de cálculos); además, todos los pacientes, después de finalizar las 3 semanas de tratamiento, fueron evaluados con tomografía computada, que no correspondió al control de imagen del nomograma del estudio de Kim porque se realizó con tomografía computada y radiografía simple de abdomen, esta última con una sensibilidad de $60 \%$, o menor, para la detección de cálculos renales y la tomografía con $98 \%$, que puede reportar mayor número de falsos negativos. A su vez, la naturaleza retrospectiva 
del estudio de Kim es una limitante, como lo determina en el análisis final.

Hasta la fecha existen pocos nomogramas disponibles; los más representativos señalan que al evaluar las variables con significación estadística reportan gran heterogeneidad y no refieren la tomografía computada como estudio de imagen durante el seguimiento, caso contario a nuestro estudio, en el que utilizamos este método en todos los pacientes, lo que dio mayor exactitud al análisis. ${ }^{22,26-28}$

En nuestro estudio la efectividad para establecer la tasa libre de cálculos fue de $69 \%$, después de evaluar a todos los pacientes con tomografía computada simple de abdomen y no con radiografía, que resulta importante mencionarlo, porque no encontramos estudios que evalúen de esta manera (en un nomograma) a los pacientes al finalizar del tratamiento. Con lo anterior definimos que la litotricia extracorpórea por ondas de choque es un método atractivo entre los diferentes tratamientos para la litiasis renal. Tomando en cuenta las variables con significación estadística de nuestro estudio, en investigaciones futuras podremos tener mayor selección de pacientes ideales para litotricia extracorpórea por ondas de choque. Esta técnica es preferible por la mayoría de los pacientes, debido a su naturaleza no invasiva, carácter ambulatorio, colocación poco frecuente de catéteres doble J y baja incidencia de complicaciones.

\section{CONCLUSIONES}

El nomograma con variables clínicas y tomográficas para la predicción de tasa libre de cálculos mostró un rendimiento por debajo de lo esperado; sin embargo, se obtuvieron datos y variables con significación estadística aplicables para la población mexicana y puede ser útil en la práctica clínica, con previo asesoramiento en pacientes ideales para litotricia extracorpórea por ondas de choque. Aunque esta técnica está dejando de utilizarse, debido al surgimiento de nuevos métodos, aún muestra alta efectividad en casos seleccionados. Se requieren nuevas investigaciones, con nomogramas que incluyan variables validadas en la población mexicana y que funcione como herramienta complementaria en la selección de pacientes para litotricia extracorpórea por ondas de choque.

\section{REFERENCIAS}

1. Türk C, et al. Guidelines on urolithiasis. EAU 2017 [en línea]. Dirección URL: http://uroweb.org/guideline/urolithiasis/

2. Wein A, et al. Campbell-Walsh Urology. $11^{\text {th }}$ ed. Philadelphia: Saunders; 2016.

3. Otero $F$, et al. Las enfermedades renales en el instituto mexicano del seguro social (1982-1989). Rev Asoc Med IntMex 1995;44 (6):21-29.

4. http://www.hgm.salud.gob.mx/descargas/pdf/area_medica/urolo/3litiasis_renal.pdf

5. Chaussy C, et al. Extracorporeal shockwave lithotripsy (ESWL): a chronology. DOI: https://doi.org/10.1089/ end.2007.9880

6. Williams J, et al. Variability of renal stone fragility in shock wave lithotripsy. Urology 2003;61(6):1092-1096.

7. Bhojani N, Lingeman J. Shockwave lithotripsy-new concepts and optimizing treatment parameters. DOI: https://doi. org/10.1016/j.ucl.2012.09.001

8. Pareek $\mathrm{G}$, et al. Shock wave lithotripsy success determined by skin-to-stone distance on computed tomography. DOI: https://doi.org/10.1016/j.urology.2005.05.011

9. Wiesenthal J, et al. Evaluating the importance of mean stone density and skin-to-stone distance in predicting successful shock wave lithotripsy of renal and ureteric calculi. Urol Res 2010;38(4):307-313.

10. Wang $L$, et al. Predictions of outcomes of renal stones after extracorporeal shock wave lithotripsy from stone characteristics determined by unenhanced helical computed tomography: A multivariate analysis. Eur Radiol 2005;15(11):2238-2243.

11. Bandi G, et al. Stone measurement by volumetric threedimensional computed tomography for predicting the outcome after extracorporeal shock wave lithotripsy. DOI: https://doi.org/10.1111/j.1464-410X.2008.08069.x

12. Jain PM, et al. Efficacy and safety of the Healthtronics LitthoTron lithotripter. DOI: https://doi.org/10.1089/ end.2004.18.P1

13. Sahinkanat $T$, et al. Evaluation of the effects of relationships between main spatial lower pole calyceal anatomic factors on the success of shock-wave lithotripsy in patients with lower pole kidney stones. DOI: https://doi.org/10.1016/j. urology.2007.11.052 
14. Preminger GM. Management of lower pole renal calculi: shock wave lithotripsy versus percutaneous nephrolithotomy versus flexible ureteroscopy. Urol res 2006;34(2):108-111.

15. Pearle $\mathrm{M}$, et al. Prospective, randomized trial comparing shock wave lithotripsy and ureteroscopy for lower pole calicial calculi $1 \mathrm{~cm}$ or less. DOI: https://doi.org/10.1097/01. ju.0000158458.51706.56

16. Manikandan R, et al. Do anatomic factors pose a significant risk in the formation of lower pole stones? DOI: https:// doi.org/10.1016/j.urology.2007.01.005

17. Hyams $E$, et al. Flexible ureterorenoscopy and holmium laser lithotripsy for the management of renal stone burdens that measure 2 to $3 \mathrm{~cm}$ : a multiinstitutional experience. https://doi.org/10.1089/end.2009.0629

18. Riley J, et al. Retrogadeureteroscopy for renal stones larger than $2.5 \mathrm{~cm}$. DOI: https://doi.org/10.1089/end.2009.0391

19. Aboumarzouk $\mathrm{O}$, et al. Extracorporeal shock wave lithotripsy (ESWL) versus ureteroscopic management for ureteric calculi. DOI: 10.1002/14651858.CD006029.pub4

20. Srisubat $A$, et al. Extracorporeal shock wave lithotripsy (ESWL) versus percutaneous nephrolithotomy (PCNL) or retrograde intrarrenal surgery (RIRS) for kidney stones. DOI: 10.1002/14651858.CD007044.pub3

21. Tailly $\mathrm{T}$, et al. Multicenter external validation and comparison of stone scoring systems in predicting outcomes after percutaneous nephrolithotomy. DOI: https://doi. org/10.1089/end.2015.0700

22. Kanao $\mathrm{K}$, et al. Preoperative nomograms for predicting stone-free rate after extracorporeal shock wave lithotripsy. DOI: https://doi.org/10.1016/j.juro.2006.06.089

23. Dhar M, Denstedt J. Imaging in diagnosis, treatment, and follow-up of stone patients. Adv chronic kidney dis. DOI: https://doi.org/10.1053/j.ackd.2008.10.005

24. Wiesenthal J, et al. A clinical nomogram to predict the successful shock wave lithotripsy of renal and ureteral calculi. DOI: https://doi.org/10.1016/j.juro.2011.03.109

25. Niwa N, et al. Simple and practical nomograms for predicting the stone-free rate after shock wave lithotripsy in patients with a solitary upper ureteral stone. World J Urol 2017;35(9):1455-1461.

26. Timothy $\mathrm{Y}$, et al. Triple $\mathrm{D}$ score is a reportable predictor of shockwave lithotripsy stone-free rates. DOI: https://doi. org/10.1089/end.2014.0212

27. Kim J, et al. Clinical nomograms to predict stone-free rates after shock-wave lithotripsy: Development and internal-validation. DOI: https://doi.org/10.1371/journal. pone. 0149333

28. Wiesenthal J, et al. A clinical nomogram to predict the successful shockwave lithotripsy of renal and ureteral calculi. European urology supplements. DOI: https://doi. org/10.1016/j.juro.2011.03.109

\section{AVISO IMPORTANTE}

La Revista Mexicana de Urología se convierte en una publicación solo digital, con todas las ventajas que los medios y dispositivos electrónicos ofrecen. Usted podrá revisar la información mediante el sitio web (www.revistamexicanadeurologia.org.mx) o descargando la app para Android o iPhone.

Para consultar el texto completo de los artículos deberá registrarse por una sola vez con su correo electrónico, crear una contraseña, indicar su nombre, apellidos y especialidad.

Esta información es indispensable para saber qué consulta y cuáles son sus intereses, y poder en el futuro inmediato satisfacer sus necesidades de información. 